\title{
Color Image Registration under Illumination Changes ${ }^{\star}$
}

\author{
Raúl Montoliu ${ }^{1}$, Pedro Latorre Carmona ${ }^{2}$, and Filiberto $\mathrm{Pla}^{2}$ \\ 1 Dept. Arquitectura y Ciéncias de los Computadores \\ ${ }^{2}$ Dept. Lenguajes y Sistemas Informáticos. \\ Jaume I University \\ Campus Riu Sec s/n 12071 Castellón, Spain \\ \{montoliu, latorre, pla\}@uji.es \\ http://www.vision.uji.es
}

\begin{abstract}
The estimation of parametric global motion has had a significant attention during the last two decades, but despite the great efforts invested, there are still open issues. One of the most important ones is related to the ability to simultaneously cope with viewpoint and illumination changes while keeping the method accurate. In this paper, a Generalized least squared-based motion estimator model able to cope with large geometric transformations and illumination changes is presented. Experiments are made on a series of images showing that the presented technique provides accurate estimates of the motion and illumination parameters.
\end{abstract}

\section{Introduction}

Image registration could be defined as the process to transform an image to match another image as correctly as possible. This process is necessary when we want to compare or to integrate the data information from the two images (see [18] for a review of image registration methods). During image acquisition of a scene, many factors intervene: the position and the distance from the camera (or sensor) to the scene, the illumination, the nature of the objects to be imaged, etc. Any change in these factors implies that the data in the corresponding images are not directly comparable. During the last few years, a special interest has emerged in relation to the need to cope with simultaneous viewpoint and illumination changes ([11], [13], [1], [2], to cite a few works). Interesting examples could be found in image databases where we can obtain images of the same place acquired with different cameras and with different acquisition conditions.

In general, the direct geometric registration problem can be solved minimizing an error function in relation to the difference in the pixel values between an image that may be called Test image and the Reference image. In particular, it can be formally written as:

$$
\min _{g} \sum_{\mathbf{q} \in \mathbb{R}}\left\|\mathcal{I}_{1}\left(\mathbf{q}_{\mathbf{i}}\right)-\mathcal{I}_{2}\left(\mathcal{G}\left(\mathbf{q}_{\mathbf{i}} ; \mathbf{g}\right)\right)\right\|^{2}
$$

\footnotetext{
* This work has been partially funded by projects AYA2008-05965-C04-04 and CSD200700018 from Spanish Ministry of Science and Innovation.
}

E. Bayro-Corrochano and J.-O. Eklundh (Eds.): CIARP 2009, LNCS 5856, pp. 61-68, 2009.

(C) Springer-Verlag Berlin Heidelberg 2009 
where $\mathcal{I}_{1}$ and $\mathcal{I}_{2}$ are two input images, $\mathbf{q}_{\mathbf{i}}=\left(x_{i}, y_{i}\right)^{T}$ are the pixel coordinates, $\mathbf{g}$ is the vector of motion parameters and $\mathcal{G}$ is the function to transform the pixel coordinates from one image to the other. The function $\mathcal{G}$ is expressed, for instance, in an affine motion (Eq.2) as follows:

$$
\mathcal{G}\left(\mathbf{q}_{\mathbf{i}} ; \mathbf{g}\right)=\left(\begin{array}{l}
a_{1} x_{i}+b_{1} y_{i}+c_{1} \\
a_{2} x_{i}+b_{2} y_{i}+c_{2}
\end{array}\right), \mathbf{g}=\left(a_{1}, b_{1}, c_{1}, a_{2}, b_{2}, c_{2}\right) .
$$

If photometric changes are also considered, these may be modeled by a transformation $\mathcal{P}$ with parameter vector $\mathbf{p}$ and the minimization would therefore be:

$$
\min _{g} \sum_{\mathbf{q} \in \mathbb{R}}\left\|\mathcal{I}_{1}\left(\mathbf{q}_{\mathbf{i}}\right)-\mathcal{P}\left(\mathcal{I}_{2}\left(\mathcal{G}\left(\mathbf{q}_{\mathbf{i}} ; \mathbf{g}\right)\right) ; \mathbf{p}\right)\right\|^{2}
$$

To solve the problem shown in Eq. 3. Bartoli developed the Dual Inverse Compositional (DIC) estimation technique [2] considering Eq. 3] and then applying an inverse compositional update rule for both the geometric and photometric transformations. See [2] for details on the steps of the algorithm used to assess the geometric registration and illumination compensation parameters.

In this paper a generalized least squares-based non-linear motion estimation technique that incorporates the capability to deal with color illumination changes is presented (it will be called the GLSIC method), where illumination changes are modeled considering an affine transformation framework. The method is based on the Generalized Least Squares (GLS) motion estimation method introduced by Montoliu and Pla in [12], and where a new criterion function is proposed, incorporating these illumination changes. The GLS method is applied on this criterion function, deriving a new set of equations whose solutions allow the simultaneous assessment of the geometric and affine illumination transformation parameters. It is shown that the proposed method provides better results than the method recently described in [2]. This method is used since it is, for the best of our knowledge, the most relevant technique that simultaneously estimates the motion and the illumination transformation parameters in color images.

The rest of the paper is organized as follows: Section 2 justifies the use of a complete affine transform to model illumination changes. In Section 3 the GLS for general problems is briefly introduced. Section 4 presents the GLSIC method. Section 5 shows the experiments and results obtained by the proposed method. Conclusions are drawn in Section 6

\section{Illumination Compensation Model}

Illumination compensation is closely related to chromatic adaptation in human colour vision. The first chromatic adaptation experiments started in the late 1940s. A few years later, Wyszecki and Stiles proved in a human asymmetric matching experiment [16] that a diagonal linear matrix transform would be enough to reproduce the experiment of asymmetric matching. However, West and Brill [15] and others proved later that for a given set of sensor sensitivities a diagonal transform could only cover a restricted group of object colours and illuminant spectra. Finlayson et al [3] argued that a diagonal transform would be enough for the modeling of an illumination change if the camera has 
extremely narrow-band sensors, which is often not the case. There are other cases where diagonal illumination compensation can fail, for instance, if there are other processes happening like bias in the camera, or saturated colours in the scene. In the latter case, some colours would fall in (and outside of) the camera gamut boundary [4]. This is the reason why the use of a complete (full) affine transform in the form $\boldsymbol{\Omega} \cdot \mathcal{I}(\mathbf{q})+\boldsymbol{\Phi}$ is justified (see, for example, [6], [9], [17], to cite a few), where $\Omega \in \mathbb{R}^{3 \times 3}$ is a full matrix, with elements $\omega_{k l},(k, l=1, \ldots, 3)$, and $\boldsymbol{\Phi} \in \mathbb{R}^{3}$, a vector with elements $\phi_{k}$, $(k=1, \ldots, 3)$.

\section{Generalized Least Squares Estimation for General Problems}

In general, the GLS estimation problem can be expressed as follows (see [12] for more details):

$$
\operatorname{minimize}\left\{\Theta_{v}=v^{T} v\right\} \text { subject to } \xi(\chi, \gamma)=0 \text {, }
$$

where:

- $v$ is a vector of $r$ unknown residuals in the observation space, that is, $v=\gamma-\tilde{\gamma}$, where $\gamma$ and $\tilde{\gamma}$ are the unperturbed and actually measured vector of observations, respectively.

- $\chi=\left(\chi^{1}, \ldots, \chi^{m}\right)^{T}$ is a vector of $m$ parameters;

- $\gamma$ formed by $r$ elements $\gamma_{i}, \gamma=\left(\gamma_{1}, \ldots, \gamma_{r}\right)^{T}$, each one is an observation vector with $n$ components $\gamma_{i}=\left(\gamma_{i}^{1}, \ldots, \gamma_{i}^{n}\right)^{T}$

- $\xi(\chi, \gamma)$ formed by $r$ elements $\xi_{i}\left(\chi, \gamma_{i}\right), \xi(\chi, \gamma)=\left(\xi_{1}\left(\chi, \gamma_{1}\right), \ldots, \xi_{r}\left(\chi, \gamma_{r}\right)\right)^{T}$, each one is, in general, a set of $f$ functions that depend on the common vector of parameters $\chi$ and on an observation vector $\gamma_{i}, \xi_{i}\left(\chi, \gamma_{i}\right)=\left(\xi_{i}^{1}\left(\chi, \gamma_{i}\right), \ldots, \xi_{i}^{f}\left(\chi, \gamma_{i}\right)\right)^{T}$. Those functions can be non-linear.

Thus, the solution of (4) can be addressed as an iterative optimization process starting with an initial guess of the parameters $\widehat{\chi}(0)$. At each iteration $j$, the algorithm estimates $\widehat{\Delta \chi}(j)$ to update the parameters as follows: $\widehat{\chi}(j)=\widehat{\chi}(j-1)+\widehat{\Delta \chi}(j)$. The process is stopped if the improvement $\widehat{\Delta \chi}(j)$ is lower than a threshold. The improvement $\widehat{\Delta \chi}(j)$ can be expressed as follows:

$$
\widehat{\Delta \chi}(j)=\left(\mathbf{A}^{T} \mathbf{Q} \mathbf{A}\right)^{-1} \mathbf{A}^{T} \mathbf{Q} \mathbf{e}
$$

where $\mathbf{Q}=\left(\mathbf{B B}^{T}\right)^{-1}$. Equation 5 can also be expressed as:

$$
\widehat{\Delta \chi}(j)=\left(\sum_{i=1 \ldots r} \mathbf{N}_{i}\right)^{-1}\left(\sum_{i=1 \ldots r} \mathbf{T}_{i},\right),
$$

with $\mathbf{N}_{i}=\mathbf{A}_{i}^{t}\left(\mathbf{B}_{i} \mathbf{B}_{i}^{t}\right)^{-1} \mathbf{A}_{i}$ and $\mathbf{T}_{i}=\mathbf{A}_{i}^{t}\left(\mathbf{B}_{i} \mathbf{B}_{i}^{t}\right)^{-1} \mathbf{e}_{i}$, where $\mathbf{B}_{i}$ is an $\mathbb{R}^{f \times n}$ matrix with elementes $b_{i}(k l)=\frac{\partial \xi_{i}^{k}\left(\widehat{\chi}(j-1), \gamma_{i}\right)}{\partial \gamma_{i}^{l}}(k=1, \ldots, f ; l=1, \ldots, n) ; \mathbf{A}_{i}$ is an $\mathbb{R}^{f \times m}$ matrix with elements $a_{i}(k l)=\frac{\partial \xi_{i}^{k}\left(\widehat{\chi}(j-1), \gamma_{i}\right)}{\partial \chi^{l}}(k=1, \ldots, f ; l=1, \ldots, m)$; and finally $\mathbf{e}_{i}$ is an $\mathbb{R}^{f}$ vector with elements $e_{i}(k)=-\xi_{i}^{k}\left(\widehat{\chi}(j-1), \gamma_{i}\right)(k=1, \ldots, f)$. 
Table 1. $\mathbf{A}_{i}$ matrix for affine motion. First part.

\begin{tabular}{|l|c|c|c|c|c|c|}
\hline Function & $\partial a_{1}$ & $\partial b_{1}$ & $\partial c_{1}$ & $\partial a_{2}$ & $\partial b_{2}$ & $\partial c_{2}$ \\
\hline$\xi_{1}\left(\chi, \gamma_{i}\right)$ & $x_{i} R_{2}^{x}$ & $y_{i} R_{2}^{x}$ & $R_{2}^{x}$ & $x_{i} R_{2}^{y}$ & $y_{i} R_{2}^{y}$ & $R_{2}^{y}$ \\
$\xi_{2}\left(\chi, \gamma_{i}\right)$ & $x_{i} G_{2}^{x}$ & $y_{i} G_{2}^{x}$ & $G_{2}^{x}$ & $x_{i} G_{2}^{y}$ & $y_{i} G_{2}^{y}$ & $G_{2}^{y}$ \\
$\xi_{3}\left(\chi, \gamma_{i}\right)$ & $x_{i} B_{2}^{x}$ & $y_{i} B_{2}^{x}$ & $B_{2}^{x}$ & $x_{i} B_{2}^{y}$ & $y_{i} B_{2}^{y}$ & $B_{2}^{y}$ \\
\hline
\end{tabular}

Table 2. $\mathbf{A}_{i}$ matrix for affine and projective motion. Second part.

\begin{tabular}{|c|c|c|c|c|c|c|c|c|c|c|c|c|}
\hline Function & $\partial \alpha_{11}$ & $\partial \alpha_{12}$ & $\partial \alpha_{13}$ & $\partial \alpha_{21}$ & $\partial \alpha_{22}$ & $\partial \alpha_{23}$ & $\partial \alpha_{31}$ & $\partial \alpha_{32}$ & $\partial \alpha_{33}$ & $\partial \beta_{1}$ & $\partial \beta_{2}$ & $\partial \beta_{3}$ \\
\hline$\xi_{1}\left(\chi, \gamma_{i}\right)$ & $-R_{1}$ & $-G_{1}$ & $-B_{1}$ & 0 & 0 & 0 & 0 & 0 & 0 & -1 & 0 & 0 \\
$\xi_{2}\left(\chi, \gamma_{i}\right)$ & 0 & 0 & 0 & $-R_{1}$ & $-G_{1}$ & $-B_{1}$ & 0 & 0 & 0 & 0 & -1 & 0 \\
$\xi_{3}\left(\chi, \gamma_{i}\right)$ & 0 & 0 & 0 & 0 & 0 & 0 & $-R_{1}$ & $-G_{1}$ & $-B_{1}$ & 0 & 0 & -1 \\
\hline
\end{tabular}

\section{GLS-Based Color Motion Estimation under Illumination Changes}

In the GLSIC formulation of the motion estimation problem, the function $\xi_{i}\left(\chi, \gamma_{i}\right)$ is expressed as follows:

$$
\xi_{i}\left(\chi, \gamma_{i}\right)=\mathcal{I}_{2}\left(\mathbf{q}_{i}^{\prime}\right)-\mathcal{P}^{-1}\left(\mathcal{I}_{1}\left(\mathbf{q}_{i}\right) ; \mathbf{p}\right)
$$

with $\mathcal{I}_{1}\left(\mathbf{q}_{i}\right)=\left(R_{1}\left(\mathbf{q}_{i}\right), G_{1}\left(\mathbf{q}_{i}\right), B_{1}\left(\mathbf{q}_{i}\right)\right)^{T}$ and $\mathcal{I}_{2}\left(\mathbf{q}_{i}^{\prime}\right)=\left(R_{2}\left(\mathbf{q}_{i}^{\prime}\right), G_{2}\left(\mathbf{q}_{i}^{\prime}\right), B_{2}\left(\mathbf{q}_{i}^{\prime}\right)\right)^{T}$, where $\mathbf{q}_{i}^{\prime}$ has been introduced to simplify notation as: $\mathbf{q}_{i}^{\prime}=\mathcal{G}\left(\mathbf{q}_{i} ; \mathbf{g}\right)$. Note that in this case the number of functions $f$ is 3 . Eq. 7 can also be writen in a more convenient way as follows:

$$
\begin{aligned}
& \xi_{i}^{1}\left(\chi, \gamma_{i}\right)=R_{2}\left(\mathbf{q}_{i}^{\prime}\right)-\left(R_{1}\left(\mathbf{q}_{i}\right) \omega_{11}+G_{1}\left(\mathbf{q}_{i}\right) \omega_{12}+B_{1}\left(\mathbf{q}_{i}\right) \omega_{13}+\phi_{1}\right) \\
& \xi_{i}^{2}\left(\chi, \gamma_{i}\right)=G_{2}\left(\mathbf{q}_{i}^{\prime}\right)-\left(R_{1}\left(\mathbf{q}_{i}\right) \omega_{21}+G_{1}\left(\mathbf{q}_{i}\right) \omega_{22}+B_{1}\left(\mathbf{q}_{i}\right) \omega_{23}+\phi_{2}\right) \\
& \xi_{i}^{3}\left(\chi, \gamma_{i}\right)=B_{2}\left(\mathbf{q}_{i}^{\prime}\right)-\left(R_{1}\left(\mathbf{q}_{i}\right) \omega_{31}+G_{1}\left(\mathbf{q}_{i}\right) \omega_{32}+B_{1}\left(\mathbf{q}_{i}\right) \omega_{33}+\phi_{3}\right)
\end{aligned}
$$

where $R_{1}\left(\mathbf{q}_{i}\right), G_{1}\left(\mathbf{q}_{i}\right)$ and $B_{1}\left(\mathbf{q}_{i}\right)$ are the $R, G$ and $B$, components of the first color image in the sequence (Reference image) at the point $\mathbf{q}_{i}$, and $R_{2}\left(\mathbf{q}_{i}^{\prime}\right), G_{2}\left(\mathbf{q}_{i}^{\prime}\right)$ and $B_{2}\left(\mathbf{q}_{i}^{\prime}\right)$ are the $R, G$ and $B$, components of the second color image in the sequence (Test image ) at the transformed point $\mathbf{q}_{i}^{\prime}=\mathcal{G}\left(\mathbf{q}_{i} ; \mathbf{g}\right)$. In this case, each observation vector $\gamma_{i}$ is related to each pixel $\mathbf{q}_{i}$, with $r$ being the number of pixels in the area of interest.

Let us define the observation vector as $\gamma_{i}=\left(R_{1}\left(\mathbf{q}_{i}\right), G_{1}\left(\mathbf{q}_{i}\right), B_{1}\left(\mathbf{q}_{i}\right), x_{i}, y_{i}\right)$. The vector of parameters is defined as follows: $\chi=(\mathbf{g}, \mathbf{p})^{T}$. Due to the high dimensionality of the parameter vector it is difficult to describe $\mathbf{A}_{i}, \mathbf{B}_{i}$ using matrix form. Tables will be used instead. For affine motion, $\mathbf{A}_{i}$ is shown in Tables 1 and $2, \mathbf{B}_{i}$ is shown in Tables 3 and 4 For projective motion, $\mathbf{A}_{i}$ is shown in Tables 5 and 2 , $\mathbf{B}_{i}$ is shown in Tables 3 and 6 .

In Tables 1 to 6 $R_{1}^{x}, R_{1}^{y}, G_{1}^{x}, G_{1}^{y}, B_{1}^{x}, B_{1}^{y}, R_{2}^{x}, R_{2}^{y}, G_{2}^{x}, G_{2}^{y}, B_{2}^{x}$ and $B_{2}^{y}$ have been introduced to simplify notation as follows: $R_{1}^{x}\left(\mathbf{q}_{i}\right), R_{1}^{y}\left(\mathbf{q}_{i}\right), G_{1}^{x}\left(\mathbf{q}_{i}\right), G_{1}^{y}\left(\mathbf{q}_{i}\right), B_{1}^{x}\left(\mathbf{q}_{i}\right)$, $B_{1}^{y}\left(\mathbf{q}_{i}\right)$ (components of the gradient of the $R, G, B$ bands of the reference image at 
Table 3. $\mathbf{B}_{i}$ matrix for affine and projective motion. First part.

\begin{tabular}{|l|l|l|l|}
\hline Function & $\partial R_{1}$ & $\partial G_{1}$ & $\partial B_{1}$ \\
\hline$\xi_{1}\left(\chi, \gamma_{i}\right)$ & $-\alpha_{11}$ & $-\alpha_{12}$ & $-\alpha_{13}$ \\
$\xi_{2}\left(\chi, \gamma_{i}\right)$ & $-\alpha_{21}$ & $-\alpha_{22}$ & $-\alpha_{23}$ \\
$\xi_{3}\left(\chi, \gamma_{i}\right)$ & $-\alpha_{31}$ & $-\alpha_{32}$ & $-\alpha_{33}$ \\
\hline
\end{tabular}

Table 4. $\mathbf{B}_{i}$ matrix for affine motion. Second part.

\begin{tabular}{|c|c|c|}
\hline Function & $\partial x$ & $\partial y$ \\
\hline$\xi_{1}\left(\chi, \gamma_{i}\right)$ & $\left(a_{1} R_{2}^{x}+a_{2} R_{2}^{y}\right)-\left(\alpha_{11} R_{1}^{x}+\alpha_{12} G_{1}^{x}+\alpha_{13} B_{1}^{x}\right)$ & $\left(b_{1} R_{2}^{x}+b_{2} R_{2}^{y}\right)-\left(\alpha_{11} R_{1}^{y}+\alpha_{12} G_{1}^{y}+\alpha_{13} B_{1}^{y}\right)$ \\
$\xi_{2}\left(\chi, \gamma_{i}\right)$ & $\left(a_{1} G_{2}^{x}+a_{2} G_{2}^{y}\right)-\left(\alpha_{21} R_{1}^{x}+\alpha_{22} G_{1}^{x}+\alpha_{23} B_{1}^{x}\right)$ & $\left(b_{1} G_{2}^{x}+b_{2} G_{2}^{y}\right)-\left(\alpha_{21} R_{1}^{y}+\alpha_{22} G_{1}^{y}+\alpha_{23} B_{1}^{y}\right)$ \\
$\xi_{3}\left(\chi, \gamma_{i}\right)$ & $\left(a_{1} B_{2}^{x}+a_{2} B_{2}^{y}\right)-\left(\alpha_{31} R_{1}^{x}+\alpha_{32} G_{1}^{x}+\alpha_{33} B_{1}^{x}\right)$ & $\left(b_{1} B_{2}^{x}+b_{2} B_{2}^{y}\right)-\left(\alpha_{31} R_{1}^{y}+\alpha_{32} G_{1}^{y}+\alpha_{33} B_{1}^{y}\right)$ \\
\hline
\end{tabular}

Input: Images $I_{1}=\left(R_{1}, G_{1}, B_{1}\right)^{T}$ and $I_{2}=\left(R_{2}, G_{2}, B_{2}\right)^{T}$

Output: $\hat{\chi}$, the vector of estimated motion parameters.

1. Calculate image gradients.

2. $j=0$.

3. Set $\Omega(0)=\mathbf{I}, \Phi(0)=(0,0,0)^{T}$ and $\mathbf{g}(0)=$ FeatureStep $\left(I_{1}, I_{2}\right)$.

4. $\widehat{\chi}(0)=(\mathbf{g}(0), \mathbf{p}(0))^{T}$, with $\mathbf{p}(0)=\left(\omega_{11}(0), \ldots, \omega_{33}(0), \phi_{1}(0), \ldots, \phi_{3}(0)\right)$.

5. repeat

6. $j=j+1$.

7. Update matrices $\mathbf{A}_{i}, \mathbf{B}_{i}$ and $\mathbf{e}_{i}$ using $\widehat{\chi}(j-1)$.

8. Estimate $\widehat{\Delta \chi}(j)$.

9. $\widehat{\chi}(j)=\widehat{\chi}(j-1)+\widehat{\Delta \chi}(j)$.

10. until $|\widehat{\Delta \chi}(j)|$ is small enough.

11. $\widehat{\chi}=\widehat{\chi}(j)$.

\section{Algorithm 1. The GLSIC algorithm}

point $\left.\mathbf{q}_{i}\right), R_{2}^{x}\left(\mathbf{q}_{i}^{\prime}\right), R_{2}^{y}\left(\mathbf{q}_{i}^{\prime}\right), G_{2}^{x}\left(\mathbf{q}_{i}^{\prime}\right), G_{2}^{y}\left(\mathbf{q}_{i}^{\prime}\right), B_{2}^{x}\left(\mathbf{q}_{i}^{\prime}\right)$ and $B_{2}^{y}\left(\mathbf{q}_{i}^{\prime}\right)$ (components of the gradient of the $R, G, B$ bands of the test image at point $\left.\mathbf{q}_{i}^{\prime}\right)$, respectively.

In addition, $N_{d}, N_{1}, N_{2}, N_{3}, N_{4}, N_{5}$ and $N_{6}$ would be:

$$
\begin{aligned}
& N_{d}=\left(d x_{i}+e y_{i}+1\right), N_{1}=a_{1} x_{i}+b_{1} y_{i}+c_{1}, N_{2}=a_{2} x_{i}+b_{2} y_{i}+c_{2} \\
& N_{3}=\frac{a_{1} N_{d}-d N_{1}}{N_{d}^{2}}, N_{4}=\frac{a_{2} N_{d}-d N_{2}}{N_{d}^{2}}, N_{5}=\frac{b_{1} N_{d}-e N_{1}}{N_{d}^{2}}, N_{6}=\frac{b_{2} N_{d}-e N_{2}}{N_{d}^{2}}
\end{aligned}
$$

The estimation process is summarized in Algorithm 1 A Feature-based Step is used to initialize the motion estimator (whenever the deformation between images is quite large a good initial vector of motion parameters is needed). It mainly consists of a SIFTbased technique [10] to detect and describe interest points, where for each interest point belonging to the first image a $K-N N$ search strategy is performed to find the k-closest interest points in the second image. Finally, for estimating the first approximation of the motion parameters a random sampling technique is used [14].

Regarding the illumination parameters at $\widehat{\chi}(0)$, they have initially been set to: $\Omega=\mathbf{I}$ and $\Phi=(0,0,0)^{T}$. 
Table 5. $\mathbf{A}_{i}$ matrix for projective motion. First part.

\begin{tabular}{|c|c|c|c|c|c|c|c|c|}
\hline Function & $\partial a_{1}$ & $\partial b_{1}$ & $\partial c_{1}$ & $\partial a_{2}$ & $\partial b_{2}$ & $\partial c_{2}$ & $\partial d$ & $\partial e$ \\
\hline$\xi_{1}\left(\chi, \gamma_{i}\right)$ & $\frac{x R_{2}^{x}}{N_{d}}$ & $\frac{y R_{2}^{x}}{N_{d}}$ & $\frac{R_{2}^{x}}{N_{d}}$ & $\frac{x R_{2}^{y}}{N_{d}}$ & $\frac{y R_{2}^{y}}{N_{d}}$ & $\frac{R_{2}^{y}}{N_{d}}$ & $\frac{-x_{i} R_{2}^{x} N_{1}-x_{i} R_{2}^{y} N_{2}}{N_{d}^{2}}$ & $\frac{-y_{i} R_{2}^{x} N_{1}-y_{i} R_{2}^{y} N_{2}}{N_{d}^{2}}$ \\
$\xi_{2}\left(\chi, \gamma_{i}\right)$ & $\frac{x G_{2}^{x}}{N_{d}}$ & $\frac{y G_{2}^{x}}{N_{d}}$ & $\frac{G_{2}^{x}}{N_{d}}$ & $\frac{G_{2}^{y}}{N_{d}}$ & $\frac{y G_{2}^{y}}{N_{d}}$ & $\frac{G_{2}^{y}}{N_{d}}$ & $\frac{-x_{i} G_{2}^{x} N_{1}-x_{i} G_{2}^{y} N_{2}}{N_{d}^{2}}$ & $\frac{-y_{i} G_{2}^{x} N_{1}-y_{i} G_{2}^{y} N_{2}}{N_{d}^{2}}$ \\
$\xi_{3}\left(\chi, \gamma_{i}\right)$ & $\frac{x B_{2}^{x}}{N_{d}}$ & $\frac{y B_{2}^{x}}{N_{d}}$ & $\frac{B_{2}^{x}}{N_{d}}$ & $\frac{x B_{2}^{y}}{N_{d}}$ & $\frac{y B_{2}^{y}}{N_{d}}$ & $\frac{B_{2}^{y}}{N_{d}}$ & $\frac{-x_{i} B_{2}^{x} N_{1}-x_{i} B_{2}^{y} N_{2}}{N_{d}^{2}}$ & $\frac{-y_{i} B_{2}^{x} N_{1}-y_{i} B_{2}^{y} N_{2}}{N_{d}^{2}}$ \\
\hline
\end{tabular}

Table 6. $\mathbf{B}_{i}$ matrix for proyective motion. Second part.

\begin{tabular}{|c|c|c|}
\hline Function & $\partial x$ & $\partial y$ \\
\hline$\xi_{1}\left(\chi, \gamma_{i}\right)$ & $\left(N_{3} R_{2}^{x}+N_{4} R_{2}^{y}\right)-\left(\alpha_{11} R_{1}^{x}+\alpha_{12} G_{1}^{x}+\alpha_{13} B_{1}^{x}\right)$ & $\left(N_{5} R_{2}^{x}+N_{6} R_{2}^{y}\right)-\left(\alpha_{11} R_{1}^{y}+\alpha_{12} G_{1}^{y}+\alpha_{13} B_{1}^{y}\right)$ \\
$\xi_{2}\left(\chi, \gamma_{i}\right)$ & $\left(N_{3} G_{2}^{x}+N_{4} G_{2}^{y}\right)-\left(\alpha_{21} R_{1}^{x}+\alpha_{22} G_{1}^{x}+\alpha_{23} B_{1}^{x}\right)$ & $\left(N_{5} G_{2}^{x}+N_{6} G_{2}^{y}\right)-\left(\alpha_{21} R_{1}^{y}+\alpha_{22} G_{1}^{y}+\alpha_{23} B_{1}^{y}\right)$ \\
$\xi_{3}\left(\chi, \gamma_{i}\right)$ & $\left(N_{3} B_{2}^{x}+N_{4} B_{2}^{y}\right)-\left(\alpha_{31} R_{1}^{x}+\alpha_{32} G_{1}^{x}+\alpha_{33} B_{1}^{x}\right)$ & $\left(N_{5} B_{2}^{x}+N_{6} B_{2}^{y}\right)-\left(\alpha_{31} R_{1}^{y}+\alpha_{32} G_{1}^{y}+\alpha_{33} B_{1}^{y}\right)$ \\
\hline
\end{tabular}

Table 7. Results of the registration using the four similarity measures

\begin{tabular}{|c|c|c|c|c|}
\hline & NAAE & NCC & ISC & SCC \\
\hline GLSIC & 0.9469 & 0.9662 & 0.7499 & 0.9648 \\
\hline DIC & 0.9341 & 0.9515 & 0.7204 & 0.9528 \\
\hline
\end{tabular}

\section{Experiments and Results}

In order to test the accuracy of the proposed motion estimation technique, several experiments were performed using a set of challenging images (some of them are shown in Fig. 1) obtained from several sources, including: Bartoli's example 1 , Brainard's examples 2 , Simon Fraser University Computational Vision Lab's examples 3 and Oxford's Visual Geometry Group's examples 4 . The first three images in the first row of Figure 1 were acquired by ourselves using a conventional digital camera and varying the illumination conditions. In all image pairs there exists a simultaneous geometric and photometric transformation. The GLSIC method was tested against the DIC method [2]. For each image pair, first, the Feature-based step was performed to obtain a good inital motion parameters vector. Then, both algorithms were applied using this initialization, obtaining two output parameters $\chi_{G L S I C}$ and $\chi_{D I C}$. With the estimated parameters, the Test image can be geometrically and photometrically transformed. Then if the parameters have been correctly estimated, the resulting images $\left(\mathcal{I}_{G L S I C}\right.$ and $\left.\mathcal{I}_{D I C}\right)$ should be very similar to the corresponding reference images.

Figure 2 shows the results obtained with the proposed technique for Bartoli's image, used in [2]. The first two images are the Test and the Reference image. The third is a panoramic image with the result of the registration. Note how the motion and the illumination parameters have been correctly estimated.

\footnotetext{
${ }^{1}$ http: //www. lasmea. univ-bpclermont.fr/Personnel/Adrien. Bartoli/ Research/DirectImageRegistration/index.html

${ }^{2}$ http: //color.psych.upenn. edu/brainard/

3 http://www.cs.sfu.ca/ c̃olour/data/objects_under_different -lights/index.html

4 http://www.robots.ox.ac.uk/ vgg/research/affine/index.html
} 

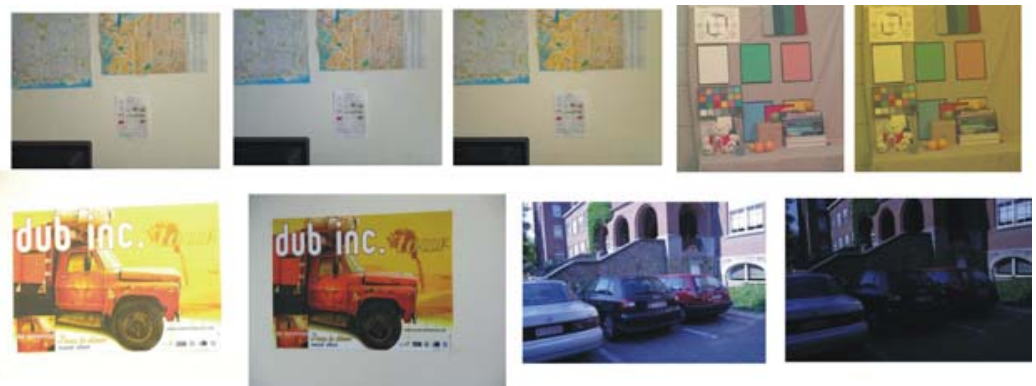

Fig. 1. Some of the images used in the experiments

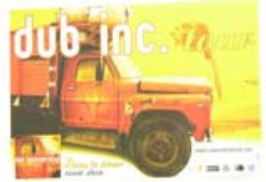

(a) Test Image

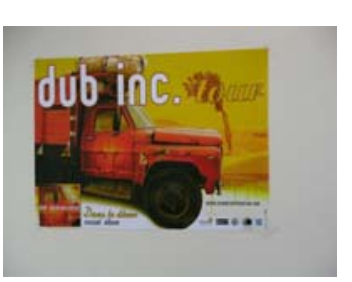

(b) Reference Image

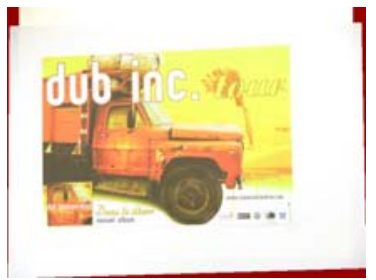

(c) Panoramic Image

Fig. 2. Registration result using Bartoli's Example in [2]

Four image similarity measures were used to assess the quality of the estimation: the Normalized Correlation Coefficient (NCC [5]), the Increment Sign Correlation coefficient ( $I S C$ [7]), the Selective Correlation Coefficient $(S C C[8])$ and the Normalized Average of Absolute Errors $(N A A E)$ defined as:

$$
N A A E(\text { aae })=\left\{\begin{array}{cc}
0 & \text { if } a a e>T H \\
\frac{T H-a a e}{T H} & \text { otherwise }
\end{array},\right.
$$

where aae is the average of the absolute errors for all the corresponding pixels in the images, and $T H$ is a constant. The four measures produce values from 0 (low similarity) to 1 (high similarity). Table 7 shows the average of the values obtained for all experiments. Note how the proposed estimation technique overcomes Bartoli's method [2] for all similarity measures.

\section{Conclusions}

In this paper, a new method able to assess the geometric and photometric transformation between image pairs has been presented. It uses a Generalized Least Squares estimation framework combined with an affine transform illumination model. It has been tested in a series of images from different sources overcoming what is considered the reference method for registration with color illumination compensation [2]. 


\section{References}

1. Bartoli, A.: Groupwise geometric and photometric direct image registration. In: Proceedings of the British Machine Vision Conference, pp. 157-166 (2006)

2. Bartoli, A.: Groupwise geometric and photometric direct image registration. IEEE Transactions on Pattern Analysis and Machine Intelligence 30, 2098-2108 (2008)

3. Finlayson, G.D., Drew, M.S., Funt, B.V.: Color constancy: generalized diagonal transforms suffice. Journal of the Optical Society of America, A 11, 3011-3019 (1994)

4. Finlayson, G.D., Hordley, S.D., Xu, R.: Convex programming colour constancy with a diagonal-offset model. In: IEEE ICIP, vol. 3, pp. 948-951 (2005)

5. Gonzalez, R.C., Woods, R.E.: Digital image processing. Prentice-Hall, Englewood Cliffs (2007)

6. Healey, G., Jain, A.: Retrieving multispectral satellite images using physics-based invariant representations. IEEE Transactions on Pattern Analysis and Machine Intelligence 18, 842848 (1996)

7. Kaneko, S., Murase, I., Igarashi, S.: Robust image registration by increment sign correlation. Pattern Recognition 35(10), 2223-2234 (2002)

8. Kaneko, S., Satoh, Y., Igarashi, S.: Using selective correlation coefficient for robust image registration. Pattern Recognition 36(5), 1165-1173 (2003)

9. Lenz, R., Tran, L.V., Meer, P.: Moment based normalization of color images. In: IEEE 3rd Workshop on multimedia signal processing, pp. 103-108 (1998)

10. Lowe, D.G.: Distinctive image features from scale-invariant keypoints. International Journal of Computer Vision 60(2), 91-110 (2004)

11. Mindru, F., Tuytelaars, T., Gool, L.V., Moons, T.: Moment invariants for recognition under changing viewpoint and illumination. Computer Vision and Image Understanding 94, 3-27 (2004)

12. Montoliu, R., Pla, F.: Generalized least squares-based parametric motion estimation. Computer Vision and Image Understanding 113(7), 790-801 (2009)

13. Shao, L., Brady, M.: Invariant salient regions based image retrieval under viewpoint and illumination variations. J. Vis. Commun. Image R. 17, 1256-1271 (2006)

14. Torr, P.H.S., Zisserman, A.: Mlesac: A new robust estimator with application to estimating image geometry. Computer Vision and Image Understanding 78, 138-156 (2000)

15. West, G., Brill, M.H.: Necessary and sufficient conditions for von kries chromatic adaptation to give color constancy. J. Math. Biol. 15, 249-258 (1982)

16. Wyszecki, G., Stiles, W.S.: Color science. John Wiley \& Sons, Chichester (1967)

17. Xingming, Z., Huangyuan, Z.: An illumination independent eye detection algorithm. In: IEEE ICPR, vol. 1, pp. 342-345 (2006)

18. Zitova, B., Flusser, J.: Image registration methods: a survey. Image and Vision Computing 21, 997-1000 (2003) 